\section{Beyond 45 Tesla}

\section{Semi-Continuous Magnetic Fields to 100 Tesla}

\author{
H. Jones \\ Department of Physics, Clarendon Laboratory, Oxford, U.K.
}

The practical and economic limit for continuous magnetic fields is $\approx 45$ tesla using hybrid magnets - a combination of stateof-the-art superconducting and watercooled solenoids energised with tens of $\mathrm{MW}$ of direct current power.

Beyond this level, non-continuous techniques are used so that the sustained massive power requirements and the consequent active cooling necessary with multi-MW magnets are not needed. Stored electrical energy from capacitor banks, flywheel generators or controlled power directly from the mains is dumped adiabatically into a coil, the thermal capacity of which is sufficient to absorb the heat without suffering a catastrophic and possibly destructive rise in temperature.

For long pulses ( $=10 \mathrm{~ms}$ to $1 \mathrm{~s}$ ), the pulse length and peak field require that the resistance of the conductor used is as low as possible. One common technique employed is to pre-cool the coil to $77 \mathrm{~K}$ or lower in order to increase the thermal capacity and reduce resistance at the start of the pulse. In Fig. 1 ladapted from Miura and Herlach [1]) a typical pulse event is shown for a coil wound from $2 \mathrm{~mm}$ cross section copper wire. The circuit includes a crowbar diode and resistor, the value of which $(0.68 \Omega)$ is designed to give maximum external energy dissipation. Also shown is the temperature rise from $77 \mathrm{~K}$. It is worth noting that at peak field, and therefore mechanical stress, the temperature is $\approx 150 \mathrm{~K}$. This has implications for the choice of conductor, as discussed below.

At peak field the solenoid experiences a maximum stress $p_{m}$ given by the magnetic pressure approximation (usually fairly conservative)

$$
p_{\mathrm{m}}=B^{2} / 2 \mu_{\mathrm{o}}
$$

where $B$ is the magnetic field and $\mu_{0}$ the permeability of vacuum. For $B=60 \mathrm{~T}, p_{\mathrm{m}}$ $=1.4 \mathrm{GPa}(14 \mathrm{kbar})$ equivalent to $\mathrm{a}$ bursting pressure some 100 times that of a standard high pressure gas cylinder, and, at $100 \mathrm{~T}$, 300 times.

Clearly these coils have to be very strong if they are not to suffer destruction at each shot. This can be achieved in a number of ways, but a fundamental requirement is a conductor which has a high mechanical strength, but at the same time low resistivity. These two properties do not normally coexist. Steel, for example, can have a tensile strength of $4 \mathrm{GPa}$, but only $1-2 \%$ the conductivity of copper, which even in its fully-hard state has only a tensile strength of $0.4 \mathrm{GPa}$. So reinforced conductors or coil constructions have to be developed.
Within Europe at present there is an initiative to design and evaluate semi-continuous high field magnets. This is the EUROMAG$\mathrm{TECH}$ consortium (University of Amsterdam, MPI Grenoble, University of Leuven, University of Oxford, INSA Toulouse) which received a European Community SCIENCE award in 1989. The objective is to explore and develop ways by which magnets can be made for fields up to $100 \mathrm{~T}$. The work includes both high strength conductors and coil construction.

\section{Coil Stresses}

For a coil with constant current density and no radial transmission of forces,

$$
B=K \sigma \underset{\max }{0.5}
$$

where $B$ is the centroid field, $\sigma_{\max }$ the maximum hoop stress and all the geometrical parameters are collected together in the constant of proportionality $K$ having a value of $1.4 \times 10^{-3}$ for a coil of say average proportions (ratio of inner and outer radii, $\alpha$, and ratio of the half-length of coil to the

If the coil is very large, i.e., $\alpha>1, \beta>1$, then $K$ becomes $2.1 \times 10^{-3}$, so the larger the coil the less the stress problem, but, of course, the greater the energy requirement for a given field. For instance, a coil of $\alpha=$ $10, \beta=3$ would require 7.5 times more energy than the $=\beta=3$ coil for the same field and bore size.

In fact, for most practical coil constructions radial stress transmission does occur, and this can modify the peak stress encountered and where it occurs. For example, the inner radius, $\beta$, both equal to 3 ). coil can be considered as a solid isotropic cylinder in which the peak stress occurs at the inner radius. Even so, equation 2 holds, but the value of $K$ ranges from $1.2 \times 10^{-3}$ for our $\alpha=\beta=3$ coil to $1.34 \times 10^{-3}$ for an infinitely large coil. The solid cylinder model can be modified by applied radia prestressing, e.g., a reinforcing cylinder on the outside of the coil. It can be shown, however, from analysis being done at present in the EUROMAGTECH programme, that this will only enhance the maximum field by $40 \%$ at most for a given strength conductor. Further gains can be made if the coil conductor is allowed to exceed its elastic limit. In such coils with radial prestress, $K \approx 3.4 \times 10^{-3}$ for a very large coil. In equation 2 , then, the constant of proportionality $K$ lies somewhere in the range $\approx 1.3 \times 10^{-3}$ to $3.5 \times 10^{-3}$, depending on construction and coil size.

Consequently, for $70 \mathrm{~T}$, conductors with yield strengths of 0.4 to $2.9 \mathrm{GPa}$ are required with conductivities a substantial fraction of copper, say $>50 \%$ IACS (International Annealed Copper Standard; $100 \%$ IACS is defined as the conductivity equal to a resistivity of $1.7241 \times 10^{-8} \Omega \mathrm{m}$ ).

\section{Conductors}

Those conductors which already exist, or are under development, and which go some of the way to fulfilling these requirements in general incorporate copper in one way or another. Obviously, the strength of the copper has to be enhanced and this results in a reduction in the conductivity. There are various copper alloys, of which the best would appear to be $\mathrm{Cu}-16$ weight $\% \mathrm{Ag}$ when the material undergoes a very high area reduction allowing extensive cold working. Tensile strengths of up to $1.5 \mathrm{GPa}$ have been achieved with conductivities of $80-90 \%$ IACS. Another approach is to use a "rule of mixtures" conductor. In this, pure copper is combined with a reinforcing material such as stainless steel.

In the EUROMAGTECH programme we have considerable experience of one such simple and economic approach, whereby a

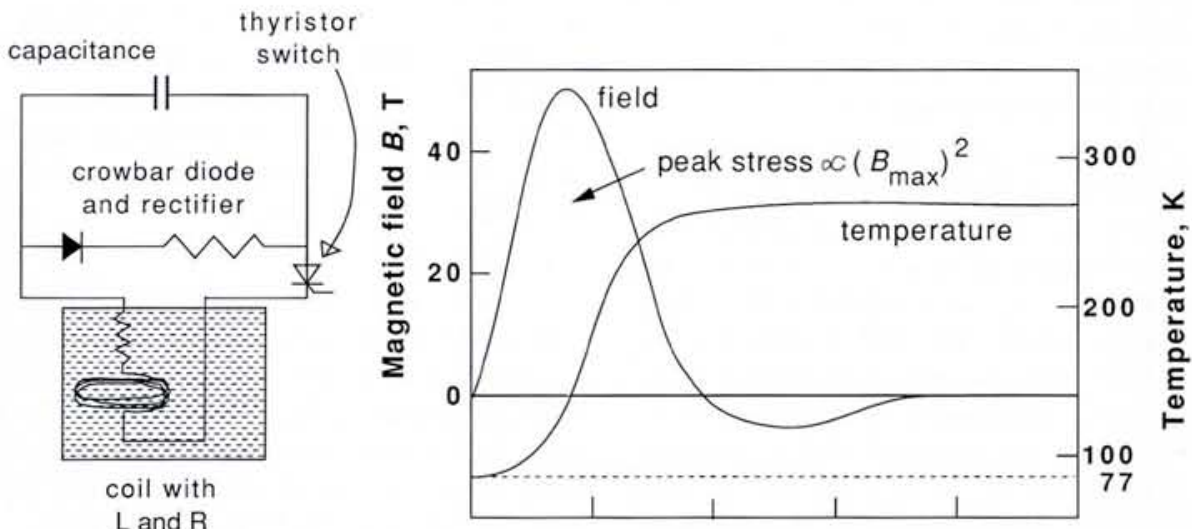

Fig. 1 - Computer simulation of a typical pulse of a small coil energised with a capacitor bank - see text. The tail on the pulse decay is characteristic of a crowbar circuit. The field strength $B$ and the temperature $T$ are plotted as a function of time $t$. 
thick-walled stainless steel tube is filled with copper and then swaged and drawn down (with the introduction of cold work) to the required size, typically $3 \times 2 \mathrm{~mm}^{2}$ [2]. Strengths in excess of $1 \mathrm{GPa}$ at room temperature have been achieved in wires with $60 \%$ cross section of copper $(=60 \%$ IACS) and such conductors have been used in small coils up to $60 \mathrm{~T}$.

One very interesting material which achieved prominence recently is the socalled micro (or nano) composite. The only commercially available version of this at the moment is produced in the USA. It is made by subjecting ingots of $\mathrm{Cu}$ with $15-20$ weight $\% \mathrm{Nb}$ to extreme cold work. $\mathrm{Cu}$ and $\mathrm{Nb}$ are mutually insoluble in the solid phase, and the $\mathrm{Nb}$ dendrites transform during the drawing process to a random array of ultrafine filaments which serve to pin the dislocations in the copper. Strengths of up to $2 \mathrm{GPa}$ with conductivities in excess of $60 \%$ IACS have been achieved in very fine wires ( $\approx 1 \mathrm{~mm}$ diameter). For the larger cross sections required for magnets, it is more difficult to achieve the necessary degree of area reduction (cold work). Rebundling and redrawing is necessary and the problems that this creates are now being addressed by a number of European industries. In the meantime, some very useful material has been produced in the USA and used in magnets for fields up to $68.4 \mathrm{~T}$ [3].

\section{Low Temperatures}

On the face of it, such microcomposites are superior to the rule of mixtures wires, but there is a complication. Earlier in this article the technique of pre-cooling to $77 \mathrm{~K}$ or lower was referred to. For some applications, therefore, the behaviour of the conductor at low temperatures is important. Table 1 compares the properties of two conductors at $300 \mathrm{~K}$ and $77 \mathrm{~K}$. They are a CuNb microcomposite and a stainless steel/ copper conductor. It can be seen that whilst the microcomposite is superior at room temperature, at liquid nitrogen temperatures the considerable increase in the strength of stainless steel coupled with the much higher $77 \mathrm{~K} / 300 \mathrm{~K}$ conductivity ratio of pure copper makes the simpler rule of mixtures conductor a strong competitor.

\section{Further Improvements}

It is expected that both types of conductors can be improved. For example, high nitrogen stainless steels can be much stronger at low temperatures, as nitrogen strengthens the martensite formed during deformation at low temperature. Billets are presently being prepared by spray deposition of high nitrogen steels onto a copper bar. It is hoped that strengths of up to $1.5 \mathrm{GPa}$ can be attained [D. Dew-Hughes, Oxford University; private communication].

Another possibility being investigated is the combination of eutectoid ( $0.8 \%$ carbon) steel wire and copper [4]. This wire has a strength of $4 \mathrm{GPa}$ when drawn to $0.25 \mathrm{~mm}$ diameter. If it were possible to combine it with $50 \%$ copper, a rule of mixtures conductor with a strength of $2 \mathrm{GPa}$ and the necessary conductivity would result.

Table 1 - Comparison between a CuNb high strength microcomposite conductor and a stainless steel/copper rule of mixtures conductor at 300 and $77 \mathrm{~K}$.

\begin{tabular}{|cccccc|}
\hline & Temperature & Proof Stress & $\begin{array}{c}\text { Ultimate } \\
\text { Tensile } \\
\text { Strength } \\
\text { GPa }\end{array}$ & Resistivity & IACS \\
\hline K & GPa & 0.722 & 1.01 & $2.40 \times 10^{-8}$ & 71 \\
\hline DCF 400 ${ }^{2}$ & 300 & 0.719 & 0.80 & $2.95 \times 10^{-8}$ & 60 \\
\hline CuNb II & 77 & 0.740 & 1.18 & $5.59 \times 10^{-9}$ & 304 \\
DCF 400 & & 1.054 & 1.23 & $3.50 \times 10^{-9}$ & 493 \\
\hline
\end{tabular}

1 CuNb $\|$ microcomposite (Cu-15 to 20 wt. \% Nb): Supercon Corp., Shrewsbury, MA, USA.

2 DCF 400 stainless steel/copper rule of mixtures composite: AEA Technology, Harwell Laboratory, UK.

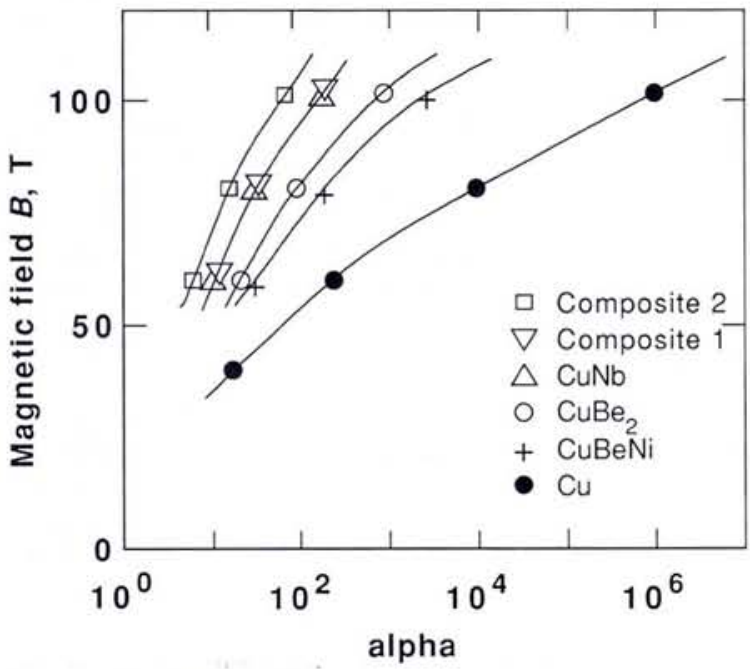

Fig. 2 - The maximum field attainable versus the size of the solenoid coil for a variety of possible conductor materials of increasing strength (after [5]). The size is given in terms of the $\alpha$, the ratio of the inner and outer radii.

Almost certainly the microcomposites can be improved. So far little work has been done on any system other than $\mathrm{CuNb}$, as this was initially developed as the precursor for manufacturing in-situ $\mathrm{Nb}_{3} \mathrm{Sn}$ superconductors. If the problems of achieving the necessary area reduction for practical size conductors, referred to earlier, can be overcome, this approach too should yield strengths up to or exceeding $2 \mathrm{GPa}$. Other possibilities include a microcomposite of $\mathrm{Cu}$ and high nitrogen stainless steel, which would exploit the excellent enhancement in strength of the steel at low temperatures. Again, suitable billets are in preparation by a number of routes.

A radical alternative to relying to such a large extent on the strength of the conductor is to wind into the solenoid a distributed reinforcement of ultra-high strength, nonconducting fibre made of "S" glass, Kevlar or carbon. This elegant technique, pioneered by $F$. Herlach and colleagues at the Catholic University, Leuven, Belgium with considerable success using simple copper wires, has achieved fields up to $67 \mathrm{~T}$ [4].

Finally, there is a method where infinite tesla can be generated regardless of the strength of the conductor, provided of course that the necessary infinite power is available. This is the so-called constant stress polyhelix design of magnet pioneered by Schneider-Muntau at the MPI, Grenoble, France [5]. In this, each layer of the solenoid is a discrete coil, the conductor section of which is adjusted so that the current density $J$ is tailored to keep the maximum hoop stress in a particular layer below the elastic limit of the conductor. Again the simple relation 2 holds. In Fig. 2, the levels of the magnetic field which can be generated are plotted as a function of a large number of values of $\alpha$. It can be seen that even harddrawn copper could be used for $100 \mathrm{~T}$, but a solenoid bore of only $20 \mathrm{~mm}$ would need an outer diameter of $20 \mathrm{~km}$ (the power requirement would be $10^{6} \mathrm{MW}$ ). More realistically, a higher strength conductor could reduce the size dramatically, e.g., Composite 1 on the figure - a maraging steel/ $50 \%$ copper rule of mixtures conductor would require an outer diameter of only $\approx 4 \mathrm{~m}$, and a power of $\approx 2000 \mathrm{MW}$. This approach is likely to be employed in the $100 \mathrm{~T}, 1 \mathrm{~s}$, "flat top" European non-continuous high magnetic field facility being discussed at present (see page 158).

\section{REFERENCES}

[1] Miura N. and Herlach F., in "Strong and Ultra Strong Magnetic Fields and Their Applications", Ed. F. Herlach (Springer, Berlin) 1985.

[2] Jones H., IEEE Trans. Magn. MAG-24 (1988) 1055.

[3] Foner S., Appl. Phys. Lett. 49 (1986) 982.

[4] Heremans G. et al., Proc. 12th Int. Conf. on Magnet Tech., Leningrad (1991), to be pub. in IEEE Trans. Magn.

[5] Schneider-Muntau H.-J., in "Megagauss Fields and Pulsed Power Systems" (Nova Science, New York) 1990. 